\title{
Increased expression of the Hutchinson-Gilford progeria syndrome truncated lamin A transcript during cell aging
}

\author{
Sofia Rodriguez ${ }^{1}$, Fabio Coppedè ${ }^{2}$, Hanna Sagelius $^{1}$ and Maria Eriksson ${ }^{* 1}$ \\ ${ }^{1}$ Department of Biosciences and Nutrition, Karolinska Institutet, Karolinska University Hospital, Huddinge, Novum, \\ Stockholm, Sweden; ${ }^{2}$ Department of Neuroscience, University of Pisa, Pisa, Italy
}

Most cases of the segmental progeroid syndrome, Hutchinson-Gilford progeria syndrome (HGPS), are caused by a de novo dominant mutation within a single codon of the LMNA gene. This mutation leads to the increased usage of an internal splice site that generates an alternative lamin A transcript with an internal deletion of 150 nucleotides, called lamin $A \Delta 150$. The $L M N A$ gene encodes two major proteins of the inner nuclear lamina, lamins $A$ and $C$, but not much is known about their expression levels. Determination of the overall expression levels of the LMNA gene transcripts is an important step to further the understanding of the HGPS. In this study, we have performed absolute quantification of the lamins $A, C$ and $A \Delta 150$ transcripts in primary dermal fibroblasts from HGPS patients and unaffected age-matched and parent controls. We show that the lamin A $\Delta 150$ transcript is present in unaffected controls but its expression is $>160$-fold lower than that in samples from HGPS patients. Analysis of transcript expression during in vitro aging shows that although the levels of lamin $A$ and lamin $C$ transcripts remain unchanged, the lamin A $\Delta 150$ transcript increases in late passage cells from HGPS patients and parental controls. This study provides a new method for LMNA transcript analysis and insights into the expression of the LMNA gene in HGPS and normal cells.

European Journal of Human Genetics (2009) 17, 928-937; doi:10.1038/ejhg.2008.270; published online 28 January 2009

Keywords: Hutchinson-Gilford progeria syndrome; laminopathy; lamin A; lamin C; lamin A 4150 ; progerin

\section{Introduction}

Hutchinson-Gilford progeria syndrome (HGPS) or progeria is a severe genetic disorder affecting about 1 per $4-8$ million live births. HGPS is classified as a segmental progeriod syndrome as multiple organs and tissues replicate phenotypes associated with normal aging. ${ }^{1}$ Children with HGPS appear healthy at birth but develop distinctive clinical features during the first years of their

${ }^{*}$ Correspondence: $\operatorname{Dr} \mathrm{M}$ Eriksson, Department of Biosciences and Nutrition, Karolinska Institutet, Karolinska University Hospital, Huddinge, Novum, Stockholm SE-14186, Sweden.

Tel: +46858583 731; Fax: +46871 166 59;

E-mail: Maria.Eriksson.2@ki.se

Received 10 September 2008; revised 11 December 2008; accepted 16 December 2008; published online 28 January 2009 life, including severe growth retardation, and loss of subcutaneous fat and skin appendages. Most children suffer from osteoporosis and die in their early teens from heart attacks and strokes caused by progressive atherosclerotic disease. ${ }^{2}$ Approximately $90 \%$ of classical HGPS is caused by a de novo mutation in exon 11 of the LMNA gene $(1824 \mathrm{C}>\mathrm{T}) .{ }^{3,4}$ The LMNA gene encodes the A-type lamins, which are intermediate filament proteins of the inner nuclear lamina. The inner nuclear lamina is functionally involved in the maintenance of nuclear shape and structure, DNA replication and gene expression. ${ }^{5}$

At least four different proteins are generated from the LMNA gene through alternative exon splicing and termination codon usage. Lamin A, encoded by exons $1-12$, and lamin $C$, encoded by exon $1-10$, are the major proteins 


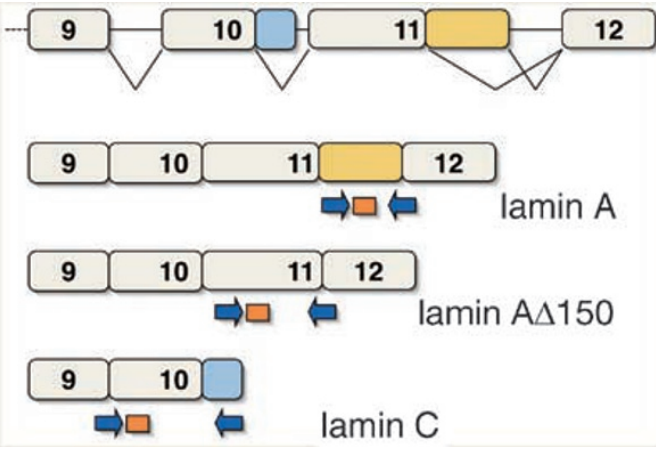

Figure 1 LMNA gene transcript quantification. Amplicons for lamins $A, A \Delta 150$ and $C$ transcript quantification located in the $3^{\prime}$ end of the LMNA gene (exons 9-12). Blue arrows and orange bars show the location of primers and TaqMan probes, respectively. The reverse primers for the lamins $A$ and $A \Delta 150$ assays, and the forward primer for the lamin $C$ assay covered exon-exon borders. The part of exon 10 specific for lamin $C$ is marked in blue. The 150 nucleotide region of exon 11, which is deleted because of alternative splicing in the lamin $A \Delta 150$ transcript is marked in yellow. Not to scale.

expressed from the LMNA locus in differentiated cells (see Figure 1). ${ }^{6,7}$ The lamin $A \Delta 10$ protein is identical to lamin A except that it lacks exon 10. Lamin $\mathrm{A} \Delta 10$ has been detected in the normal colon and a variety of carcinoma cell lines. ${ }^{8}$ Lamin C2 has an alternative exon 1 compared with lamin $\mathrm{C}$, and is present in germ cells. ${ }^{9}$ In contrast to lamins $\mathrm{C}$ and $\mathrm{C} 2$, the lamins $\mathrm{A}$ and $\mathrm{A} \Delta 10$ proteins contain CAAX boxes at their C-terminal ends, which undergo farnesylation and other post-translational modifications to become mature proteins. ${ }^{10}$

In addition to HGPS, there are 10 other different genetic disorders caused by mutations in the LMNA gene, and these disorders are collectively named laminopathies. ${ }^{11}$ The majority of the laminopathies are caused by substitutions located in exons shared by all A-type lamins (LMNA exons 2-9; for a collection of $L M N A$ variants, see the Leiden Muscular Dystrophy pages: http://www.dmd.nl). The most common HGPS mutation $(1824 \mathrm{C}>\mathrm{T})$ is located in exon 11, and therefore only affects the lamin A protein isoforms. This mutation leads to the activation of a cryptic splice donor site that removes 150 nucleotides from exon 11. The resulting lamin $\mathrm{A} \Delta 150$ mRNA gives rise to a lamin A isoform containing an internal deletion of 50 amino acids, referred to as lamin $\mathrm{A} \Delta 50$ or progerin., ${ }^{3,4}$ This truncated lamin A protein is improperly processed because of the lack of a proteolytic cleavage site for the prelamin A processing enzyme, Zmpste24. In wild-type cells, Zmpste24 removes 15 amino acids from the $C$ terminus, including a farnesylated and carboxymethylated cysteine. ${ }^{12}$ In the absence of this cleavage site, this abnormal processing leads to the generation of a stably farnesylated and carboxymethylated lamin A protein isoform, referred to as progerin..$^{13}$ In HGPS, the expression of progerin leads to irregular shaped nuclei, DNA repair defects, loss of heterochromatin, changes in histone methylation, downregulation of nuclear proteins and interferes with the onset and progression of cytokinesis. ${ }^{13-18}$

It is not clear how HGPS relates to normal physiological aging, but the identification of mutations in the LMNA gene has increased interest in the search for the role of the lamin proteins in the aging process. Studying HGPS cells, Goldman et al ${ }^{14}$ showed that nuclear defects accumulate as the cells become older in culture, which is accompanied by an increase in the amount of progerin. Progressive alterations in nuclear architecture also accompany aging in the nematode, Caenorhabditis elegans. ${ }^{19}$ Scaffidi and Misteli ${ }^{20}$ showed that cells from normal individuals of old age have similar defects compared with to those reported in cells from HGPS patients. This study also showed that the cryptic splice site, which is active in HGPS cells, is also used in cells and tissues from both young and old healthy individuals, and inhibiting the splice site in cells from old individuals reversed their nuclear abnormalities. ${ }^{20}$ Additional evidence implicating progerin in the normal aging process comes from recent reports that show that the number of progerin protein positive cells increase with age, both in vitro and in vivo, in samples from apparently healthy individuals. ${ }^{18,21}$

Cellular research in aging has been stimulated by the observation that human diploid cells have a limited number of cell divisions in culture, and therefore an analysis of cell cultures may provide valuable tools for studying the molecular mechanism of physiological senescence. ${ }^{22}$ In addition, isolated fibroblasts obtained from patients affected with premature aging syndromes, such as HGPS, show a markedly reduced life span when grown in culture. ${ }^{23}$ Determination of the overall expression levels of the $L M N A$ gene is an important step to further understanding of the progeria disease. In this study, we have developed a method for absolute quantification of LMNA locus transcripts (lamins A, A $\Delta 150$, and C). We utilized this method on RNA extracted from different passaged cells of HGPS affected individuals and controls to study changes during in vitro cell aging. Our data indicate that although the abundance of lamins A and C transcripts was unchanged, lamin $\mathrm{A} \Delta 150$ transcripts increased during in vitro cell aging in both HGPS and unaffected parental control cells.

\section{Materials and methods}

Cell cultures and growth conditions

Primary dermal fibroblasts were obtained from the Coriell Cell Repositories (Camden, NJ, USA). Selection of HGPS cultures was according to confirmed G608G mutation and stock availability. ${ }^{4}$ The HGPS cultures were AG06917, AG06297, AG03198, AG03199, AG03513, AG11513, AG11498, AG01972, and GM01972. The agematched control cultures were GM00499, GM00316, and GM01651. HGPS parent control cultures were AG06299, AG03257, AG03258, and AG03512. In some cases, multiple 
Table 1 Primary dermal fibroblasts for the quantification of LMNA locus transcripts

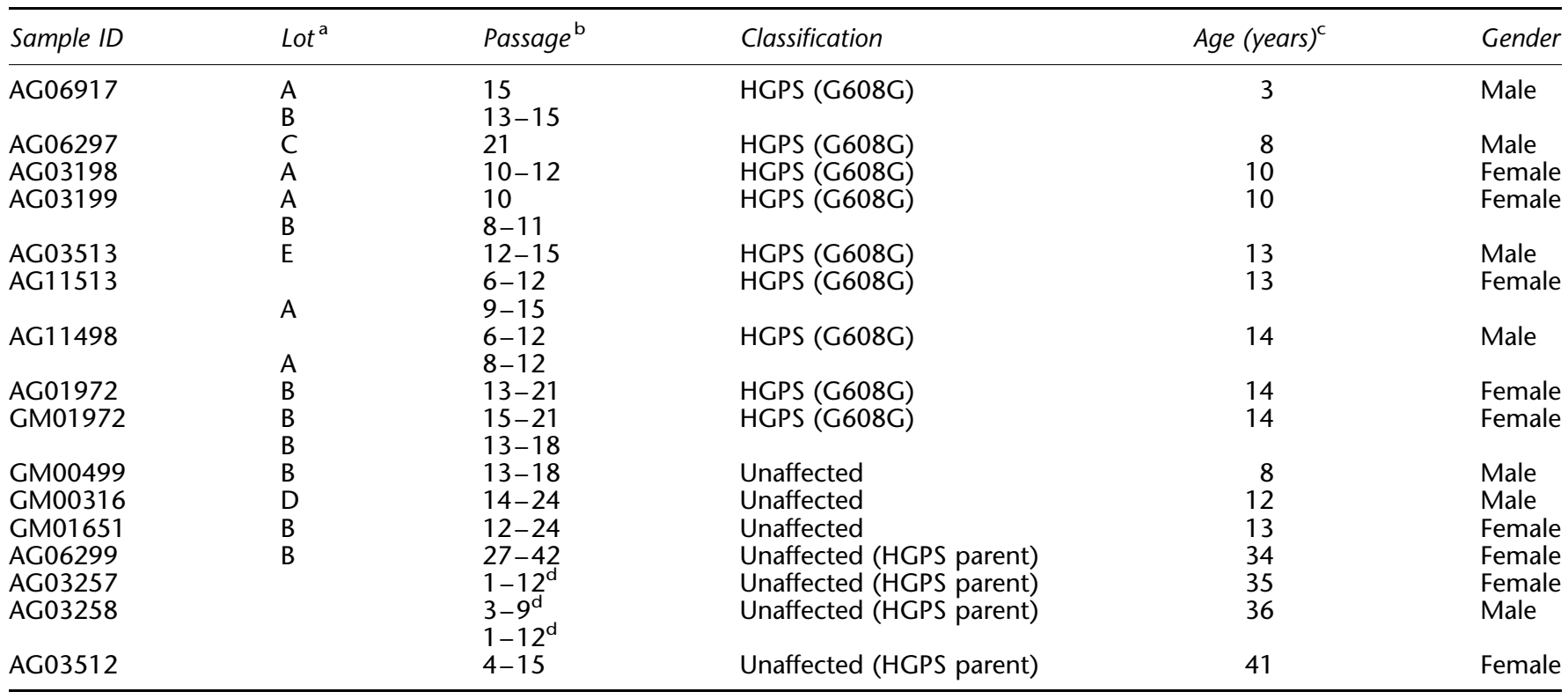

a Lot specified by Coriell cell repositories (CCR). Two lots from the same sample ID represent cultures ordered in different years and organized with the first ordered culture first and the most recent ordered culture second.

bassage or span of passages that was used for transcript quantification.

${ }^{\mathrm{C}}$ Age of patient when skin biopsy was obtained.

${ }^{\mathrm{d}}$ The number of times the cell culture was subcultured at CCR was not determined.

cultures and/or lots from the same donor were included (see Table 1). Cells were grown in a monolayer in T25 flasks (Sarstedt) in a humidified $37^{\circ} \mathrm{C} 5 \% \mathrm{CO}_{2}$ incubator. Growth medium was minimal essential medium + earle's salt (Gibco) supplemented with $15 \%$ fetal bovine serum (Biowest), and $2 \mathrm{~mm}$ L-glutamine and $1 \times$ penicillinstreptomycin (Gibco). Cells were split 1:4 on reaching confluency.

\section{RNA isolation and cDNA synthesis}

Total RNA was extracted from confluent T25 flasks of primary dermal fibroblasts using $2.5 \mathrm{ml}$ of Trizol, according to standard procedure (Invitrogen). RNA pellets were dissolved in $70 \mu \mathrm{l}$ of nuclease-free water (Ultrapure, Gibco), quantified using a spectrophotometer (BioPhotometer, Eppendorf), and treated with DNase I, according to the manufacturer's recommendations (RQ1 RNase-Free DNase, Promega). DNase was removed using RNeasy mini columns (Qiagen). $0.5 \mu \mathrm{g}$ RNA was reverse transcribed with random hexamers using the SuperScript ${ }^{\mathrm{TM}}$ cDNA Synthesis Kit (Invitrogen). cDNA samples were diluted in nuclease-free water to a final volume of $150 \mu \mathrm{l}$ and stored at $-80^{\circ} \mathrm{C}$.

\section{Taqman $^{\circledR}$ assays}

Primer and Taqman MGB probe sets were designed using Primer Express ${ }^{\circledR}$ Software version 2.0. (Applied Biosystems) and Primer $3 .^{24}$ One primer per amplicon was designed to cover an exon-exon boundary (Figure 1). Primer sequences were lamin A (exons 11/12), 5'-TCTTCTGCCTC CAGTGTCACG-3' and 5'-AGTTCTGGGGGCTCTGGGT-3'; lamin C (exons 9/10), 5'-CAACTCCACTGGGGAAGAA GTG-3' and 5'-CGGCGGCTACCACTCAC-3'; lamin A $\Delta 150$ (exons 11/12), 5'-ACTGCAGCAGCTCGGGG-3' and $5^{\prime}$-TC TGGGGGCTCTGGGC-3'. Taqman MGB probe sequences were lamin A (exon 11), 5'-ACTCGCAGCTACCG-3'; lamin C (exon10), 5'-ATGCGCAAGCTGGTG-3'; lamin A $\Delta 150$ (exon 11), 5'-CGCTGAGTACAACCT-3'. Reporter and quencher dyes for the LMNA locus assays were $5^{\prime}-6 \mathrm{FAM}^{\mathrm{TM}}$ and 3'- nonfluorescent quencher dye (NFQ; Applied Biosystems). Amplicon sizes were lamin A, 139 base pairs (bp); lamin C, 123 bp; lamin A $\Delta 150,123$ bp. Real-time PCR reactions were performed in a $20 \mu \mathrm{l}$ reaction volume, including $5 \mu \mathrm{l}$ of template, in MicroAmp 96-well plates sealed with optical adhesive covers (Applied Biosystems). Each plate contained triplicates of cDNA samples, no template controls, and a minimum of six different dilutions of cloned standards. All assays were performed in a $1 \times$ Taqman Universal master mix (Applied Biosystems). The concentration of oligos was initially optimized for each amplicon and the same batch of primers (Medprobe), and probes were used throughout this study. For lamin A, $300 \mathrm{~nm}$ forward primer, $900 \mathrm{nM}$ reverse primer, and $200 \mathrm{~nm}$ MGB probe were used. For lamin C; $300 \mathrm{~nm}$ forward primer, $900 \mathrm{nM}$ of reverse primer, and $200 \mathrm{nM} \mathrm{MGB}$ probe were used. For lamin A $\Delta 150,50 \mathrm{~nm}$ forward primer, $300 \mathrm{~nm}$ reverse primer, and $200 \mathrm{~nm}$ MGB probe were used. 
For endogenous controls, we utilized validated assays for $\beta$-glucuronidase (GUSB) and ribosomal protein large PO (RPLPO) with $\mathrm{VIC}^{\circledR}$ and NFQ as reporter and quencher dyes, respectively (Applied Biosystems). Immediately after preparation, the plates were loaded on an ABI7500 fast system sequence detection instrument and the cycling conditions were $2 \mathrm{~min}$ at $50^{\circ} \mathrm{C}, 10 \mathrm{~min}$ at $95^{\circ} \mathrm{C}$, then 50 cycles each of $15 \mathrm{~s}$ at $95^{\circ} \mathrm{C}$ and $40 \mathrm{~s}$ at $60^{\circ} \mathrm{C}$.

\section{Standard curves}

The amplicons (lamins A, C, and A $\Delta 150, \beta$-glucuronidase, and RPLPO) were amplified using standard PCR conditions from human cDNA prepared from EBV-transformed lymphoblastoid cell lines, AG03506 or AG03259 (Coriell Cell Repositories, Camden, NJ, USA). The PCR products were cloned into a pCR 2.1 vector (TOPO TA-cloning Kit, Invitrogen). The clones were purified using the Qiagen plasmid purification kit followed by a phenol-chloroform extraction and an ethanol precipitation. Plasmids were resuspended in nuclease-free water and sequenced to check that the insert had the correct sequence, and was only present in one copy. In addition, PCR using vector primers and restriction digest with EcoR1 confirmed single copy integration. Plasmid concentrations were determined using spectrophotometry (BioPhotometer, Eppendorf) and purity was confirmed by agarose gel electrophoresis. Purified plasmids were diluted in nuclease-free water and stored in single-use aliquots at $-80^{\circ} \mathrm{C}$. The same diluted plasmid preparations were used throughout this study. The standard dilutions ranged between 11 and $1.1 \times 10^{7}$ estimated copies of plasmids.

\section{Data analysis}

The fluorescence data were analyzed using the Sequence Detector System (SDS) Software 1.3.1 (Applied Biosystems). Amplification plots were analyzed by the automatic baseline option and fixed threshold values for each amplicon. Data were only considered acceptable if the correlation coefficient of the standard curve, with a minimum of five standards, exceeded 0.99, and the $C_{t}$ values did not differ by $>0.3$ units among triplicates for $C_{\mathrm{t}}$ values $<30$, and not by $>0.5$ units for $C_{t}$ values $>30$ units. $^{25}$ All three LMNA locus transcripts were normalized to the endogenous control $\beta$-glucuronidase in $1 \mu \mathrm{g}$ of total RNA. The transcript copy numbers for each of the LMNA locus transcripts were normalized to the transcript copy number for $\beta$-glucoronidase by dividing the mean of the triplicates for each LMNA locus transcript by the mean of the triplicates of $\beta$-glucoronidase for each individual sample.

\section{Statistical analysis}

All samples were assumed to be from normally distributed populations. One-way ANOVA or repeated measurement ANOVA was used to compare three independent or dependent groups, respectively. Significantly different means were post-tested using Tukey's multiple comparison test. The paired student's $t$-test, two-tailed, was used for statistical analysis of difference between early and late passages. First, normalized mean transcript levels for individual cell cultures and batches were calculated. Second, mean transcripts for each individual were calculated and used to represent normalized mean transcript values per individual (illustrated in Figure 2). Mean values

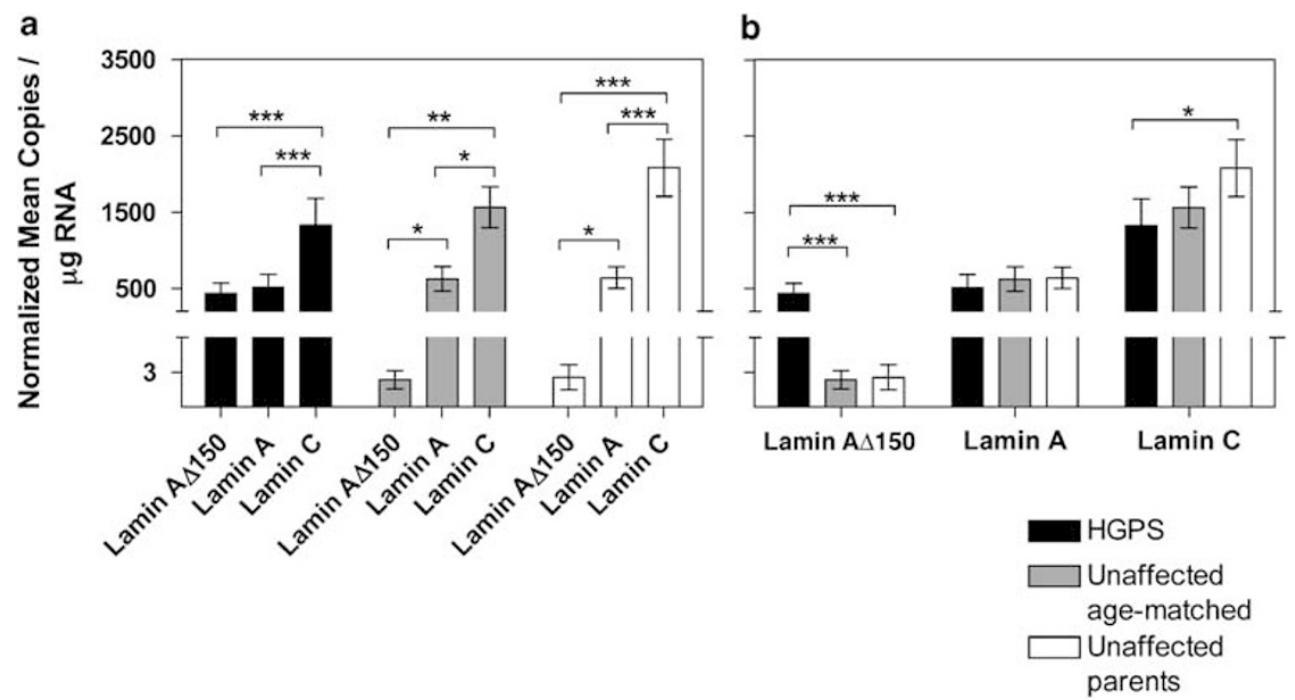

Figure 2 Absolute quantification of lamin $A \Delta 150, A$ and $C$ transcripts in primary fibroblast from HGPS patients and unaffected controls. Same data were plotted in (a) and (b), and results represent means \pm SD. RNA was extracted from different passages of primary dermal fibroblasts from seven Hutchinson-Gilford progeria syndrome patients (HGPS, $n=34$ ), three unaffected age-matched $(n=12)$, and four unaffected parent controls $(n=24)$. Asterisk represent significant differences: ${ }^{*} P<0.05,{ }^{* *} P<0.01$, and ${ }^{* * *} P<0.001$. 
of early and late passages were calculated from all cell cultures where data from more than one passage were available (first per culture, second per individual; $n=13$ ). For the HGPS sample group $(n=6)$, the early and late passages for RNA extraction ranged between passages 6-15 and 11-21, respectively. For the age-matched control group $(n=3)$, the early passage group included RNA from passage $12-13$, and the late passage group included RNA from passage $18-24$. For the adult control group $(n=4)$, the early passages included RNA from passages $4-27$, and late passages included RNA from passage 15-42. However, as the record of earlier passaging was not available from the Coriell cell repositories for some of the cultures in the adult control group, the actual range of passages might be wider (Table 1).

\section{RT-PCR analysis of LMNA splice forms}

cDNA samples from HGPS samples and unaffected controls were tested for alternative $L M N A$ splice forms. Expression of alternative lamins $\mathrm{A}$ and $\mathrm{C}$ transcript isoforms were analyzed using forward primers in exon 1, 5'-ACCTGCAGG AGCTCAATGAT-3', exon 2, 5'-CAAGAAGGAGGGTGACC TGA-3,' and exon 3, 5'-ACCATGAAGGAGGAACTGGA-3,' combined with a reverse primer in exon 12 (for lamin A), 5'-GTCCCAGATTACATGATGC-3' or exon 10 (for lamin C), 5'-CGGCGGCTACCACTCAC-3'. Additional forward primers for amplification of lamin A and/or $C$ were exon $7 / 8$, 5'-GCAACAAGTCCAATGAGGACCA-3'; exon 8/9, 5'-AGGT GGTGACGATCTGGG-3'; and exon 9/11, 5' ${ }^{\prime}$-ACTCCACTGG GGAAGGCTC-3'.

\section{Protein analysis}

Cells were collected from confluent T25 flasks, washed in PBS and lysed in RIPA buffer (including a cocktail of proteinase inhibitors (Roche)). Samples were stored at $-80^{\circ} \mathrm{C}$. Before loading, samples were thawed on ice and diluted in $16 \mathrm{M}$ of urea to a final concentration of $1.3 \mathrm{M}$ in a $5 \times$ SDS loading buffer (including freshly added $\beta$-mercaptoethanol). The samples were denatured at $100^{\circ} \mathrm{C}$ for $5 \mathrm{~min}$. Enhanced protein separation was accomplished using Protean II xi cell (Biorad) according to the previously described procedure. ${ }^{26}$ To test for changes in protein levels during in vitro aging, protein extracts from multiple passages from the same culture were loaded on the same gel. For the HGPS sample group $(n=6)$, the early and late passages for protein extraction included passages 6-12 and 9-18, respectively. For the age-matched and adult unaffected control group $(n=6)$, the early and late passages for protein extraction included passages $1-27$ and 6-42, respectively. As noted above, the actual range of passages may be wider. Primary antibodies used for western blot were mouse monoclonal antihuman lamin $\mathrm{A} / \mathrm{C}$ (mab3211, Chemicon), goat polyclonal antihuman lamin A/C (sc-6215, Santa Cruz Biotechnology), mouse monoclonal antihuman lamin A (ab8980, Abcam Ltd) and rabbit antihuman lamin C (BP4505, Acris antibodies). All blots were reincubated with antiactin (mouse monoclonal anti$\beta$-actin, A5441, Sigma), which was used for normalization. Protein quantification was performed on western filters by densitometry using the Versa Doc Imaging system (Biorad) and analyzed with the Quantity One software (Biorad). The ratio of the normalized protein value from late passage of a culture to the normalized protein value from early passage obtained from the same culture was used to analyze changes in protein quantities with increased passages between the HGPS sample group and the unaffected control group. Statistical analysis was performed using the two-tailed unpaired student's t-test. To test for differences with aging, a one-sample $t$-test comparing the mean ratio for each protein and sample group to 1 was performed.

\section{RESULTS}

\section{Validation of TaqMan assays}

RNA was extracted at different passages from primary dermal fibroblasts from HGPS patients and controls (Table 1). Taqman assays for absolute quantification of lamins $\mathrm{A}, \mathrm{A} \Delta \mathbf{1 5 0}$, and $\mathrm{C}$ transcripts were designed (Figure 1), and conditions were optimized using different amounts of primers and probe. The combination with the highest $\Delta \mathrm{Rn}$ and the lowest $C_{\mathrm{t}}$ was considered to be the optimal assay. Agarose gel electrophoresis of PCR products from the cDNA template confirmed the specificity of the assays with a single band of expected length. There was no PCR product generated when genomic DNA, no RT, and no template controls were tested. Plasmids containing a single copy of the amplicon of correct identity were used for the construction of standard curves.

Endogenous controls were initially selected based on low inter-sample variability on RNA microarrays with human fibroblast cultures (data not shown), and earlier usage in quantitative RT-PCR experiments on HGPS cell lines. ${ }^{17,27}$ The selected endogenous controls, $\beta$-glucuronidase, and RPLPO were evaluated on our sample material $(n=67)$, and the absolute values for estimated copies were compared. Similar expression profiles were obtained with both endogenous controls (data not shown); but as the expression level of $\beta$-glucuronidase was closer to the levels of the $L M N A$ locus transcripts, it was used for normalization.

\section{LMNA locus transcript expression in HGPS and control dermal fibroblasts}

Real-time RT-PCR of LMNA locus transcripts revealed expression of all three splice forms in all cDNA samples tested. As our approach used absolute quantification based on individual standard curves, we were able to compare different transcripts' expression levels within and between different samples (Figure 2). Comparison of the normalized mean copies of lamins A, C, and A $\Delta 150$ 
showed that lamin $\mathrm{C}$ was the most highly expressed transcript in all the sample groups (Figure 2a). In contrast to the HGPS sample group, where the lamin A and $\mathrm{A} \Delta 150$ transcripts were observed to be at a similar level (normalized mean transcript of 516 and 439 copies/ $\mu$ g RNA, respectively), the levels of the lamin A transcripts were $>240$-fold higher than the lamin A $\Delta 150$ transcripts in the unaffected age-matched and parent control groups (normalized mean lamin A transcript copies were 627 and 644 copies/ $\mu$ g RNA, compared with the normalized mean lamin $\mathrm{A} \Delta 150$ transcript copies of 2.4 and $2.6 / \mu \mathrm{g}$ RNA for the age-matched and parent control groups, respectively; Figure 2a).

Comparison of the normalized mean transcript levels of each transcript between the different sample groups (Figure 2b) showed that although there was no significant difference in the levels of lamin A transcripts, the levels of lamin $\mathrm{A} \Delta 150$ differed significantly between the different sample groups (Figure $2 \mathrm{~b}$ ). As expected, the lamin A $\Delta 150$ transcript levels were higher, $>160$-fold, in the HGPS group compared with the control groups (HGPS (mean 439 copies/ $\mu$ g RNA) vs unaffected agematched group (mean 2.4 copies/ $\mu$ g RNA), $P<0.001$, and HGPS vs unaffected parent controls (mean 2.6 copies/ $\mu$ g RNA), $P<0.001)$. However, there was no significant difference in lamin $\mathrm{A} \Delta \mathbf{1 5 0}$ transcript levels between the unaffected age-matched and parent control groups $(P>0.05)$.

Comparison of the lamin C transcript levels between the different sample groups revealed a significant difference in the mean normalized levels between the HGPS and the unaffected parental control group $(P<0.05$; Figure $2 b)$. However, there was no difference between the HGPS sample and the age-matched control groups in the level of lamin $\mathrm{C}$ transcripts $(P>0.05)$. This might indicate that lamin $\mathrm{C}$ expression is upregulated with age. However, comparison of lamin C levels between the differently aged control groups did not show any significant difference $(P>0.05$; Figure $2 b)$.

\section{Lamin A 150 transcript level increases with in vitro cell aging}

To analyze the changes in expression associated with in vitro cell aging, we calculated the mean expression of each transcript in each sample from early and late passages (Figure 3). Only cultures where multiple passages were collected for RNA extraction were included. A comparison of lamin A $\Delta 150$ transcripts in early passage HGPS cells with late passage HGPS cells revealed a significantly higher expression in later passages $(P=0.047$; Figure $3 a)$. A significantly higher expression of lamin $A \Delta 150$ transcripts was also seen in late passage cells from unaffected adult controls, when compared with early passage cells of the same control group $(P=0.007$; Figure 3a). No other significant difference was seen when
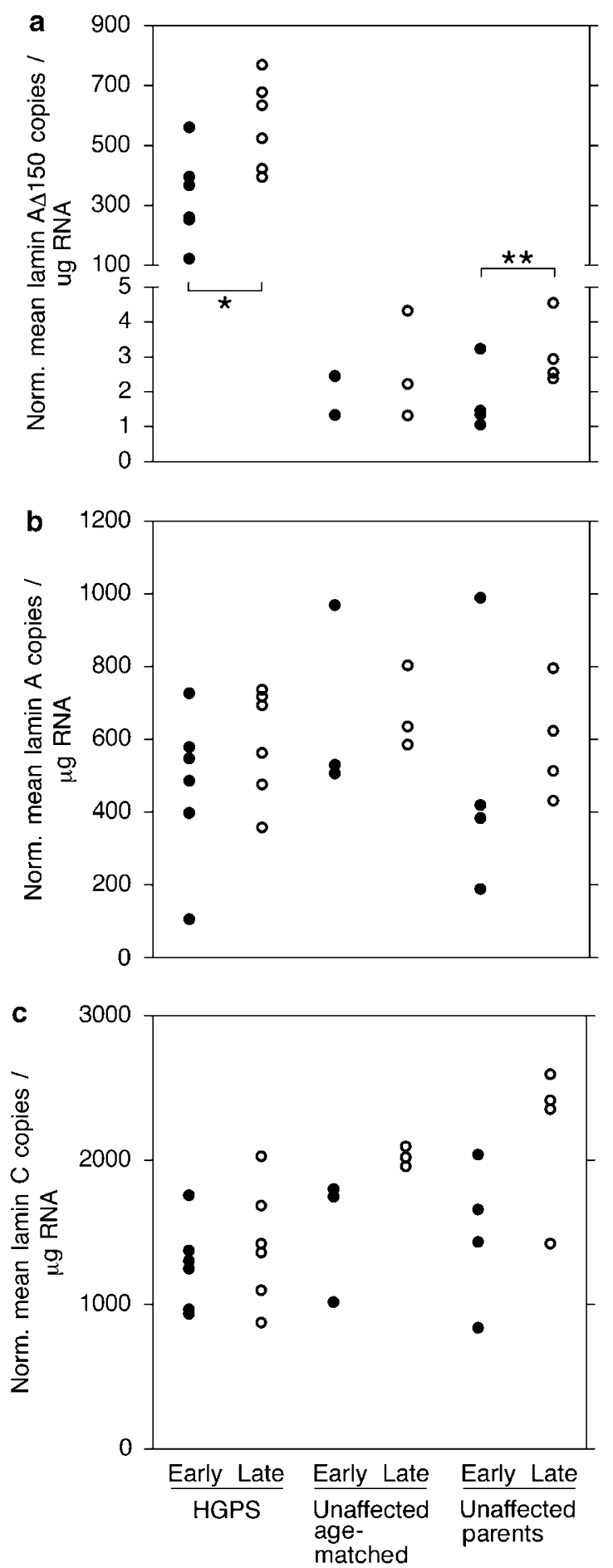

Figure $3 L M N A$ locus transcripts in early and late passage cells. Results represent means and $\pm S D$. Asterisk represent significant differences: ${ }^{*} P<0.05,{ }^{* \star} P<0.01$.

analyzing differences in the expression of $L M N A$ locus transcripts between early and late passages for the different sample groups (Figure $3 \mathrm{~b}-\mathrm{c}$ ). 


\section{Lamin A/C protein isoforms}

To test whether the changes in the RNA level were also evident at the protein level, protein was extracted from HGPS and unaffected control cells of different passages. Western blots with antibodies to human lamin A/C were used to study lamin $\mathrm{A}$ and $\mathrm{C}$ protein isoform expression during passaging (Figure 4). Enhanced protein separation revealed proteins corresponding in size to prelamin A, lamin $\mathrm{A}$, progerin, and lamin $\mathrm{C}$, when hybridized with antibodies to human lamin $\mathrm{A} / \mathrm{C}$ (Figure $4 \mathrm{a}$ right top panel, and $4 \mathrm{c}$ ). In addition, one extra fragment migrating between progerin and lamin $\mathrm{C}$ was seen in protein extracts from all individuals (HGPS and controls) (Figure 4c). The extra fragment was also seen when blots were hybridized with a lamin C-specific antibody (Figure 4a middle top panel).
In accordance with the unknown fragment being an isoform of lamin $\mathrm{C}$, the extra fragment was not seen when filters were hybridized with the lamin A-specific antibody (Figure 4a left top panel). To elucidate whether the extra fragment seen on the western blots corresponded to novel LMNA splicing event, we performed an RT-PCR analysis of the $L M N A$ gene. RT-PCR analysis using forward primers located in exons $1,2,3$, spanning the junction of exons 7 and $8(7 / 8)$, and $8 / 9$, and reverse primers in the region of exon 10 specific to lamin C, or exon 12 (specific for lamin A) did not reveal any novel LMNA splice form that could explain the extra fragment seen on western blot (Figure $4 \mathrm{~b}$, data not shown). RT-PCR products corresponding in size to the expected fragments for lamins $\mathrm{A}$ or $\mathrm{C}$ were present in all tested cDNA samples. In cDNA samples from HGPS patients,

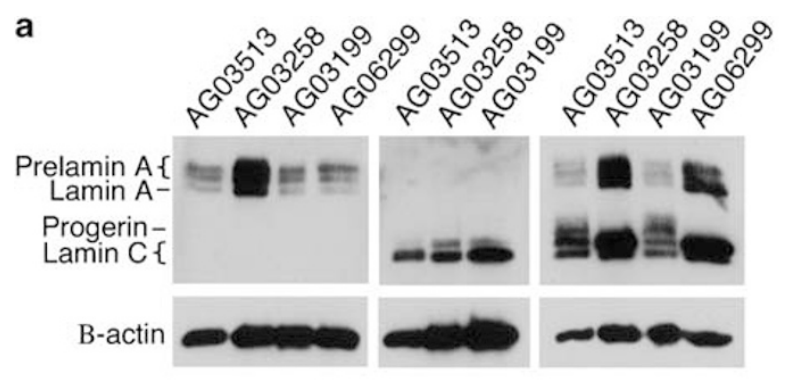

b

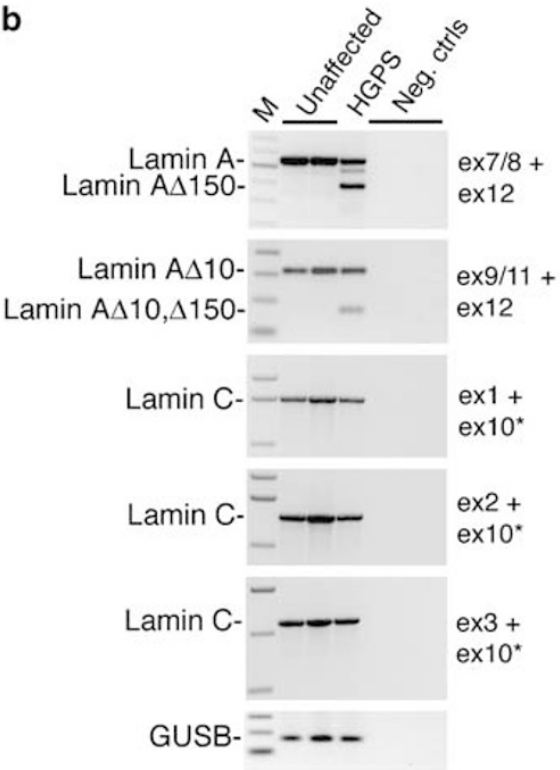

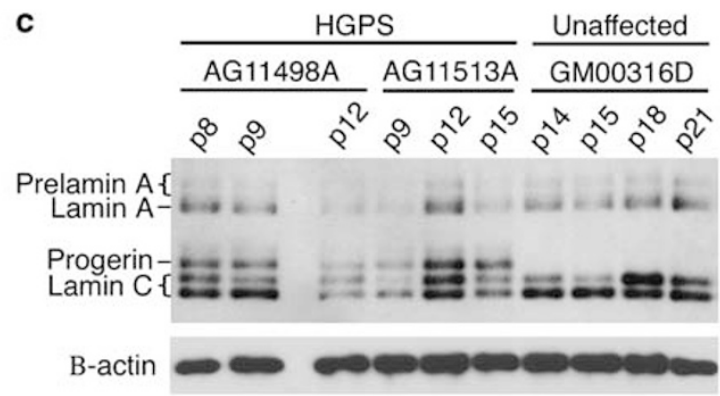

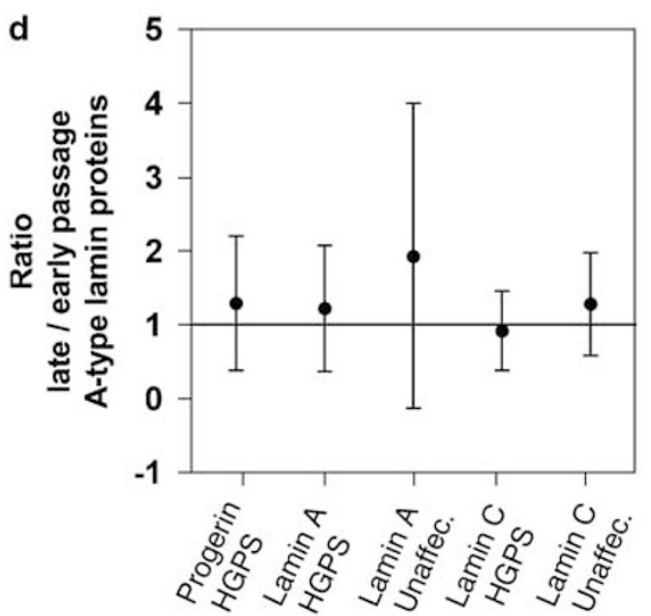

Figure 4 A-type lamins in primary fibroblasts from HGPS and unaffected controls. (a) Western blot experiments with antibodies to lamins A (left panel), C (middle panel), and A/C (right panel). (b) RT-PCR screening with exon (ex) $7 / 8$ or ex9/11, and ex12 primer showed fragments corresponding to lamins A (640 base pairs, bp) and A $\Delta 150$ (490 bp), or lamins A $\Delta 10$ (319 bp) and A $\Delta 10, \Delta 150$ (169 bp), respectively. RT-PCR with primers in ex1,2, or 3, and ex10, resulted in fragments corresponding to lamin $C(1612,1350$, and 1118 bp, respectively). Asterisk (*) indicates that the primer was located in the region of exon 10 specific for lamin C. Negative control lanes contained PCR samples with genomic DNA, no RT, and no template controls (M, 100 bp DNA ladder (Invitrogen)). (c) Representative western blot with protein extracts from multiple passages of fibroblast cell cultures. (d) Changes in protein levels during passaging illustrated as the ratio of normalized band intensities for late and early passage protein extracts of the same culture. Results represent means \pm SD. 
there was an additional band corresponding to lamin A $\Delta 150$ whenever the reverse primer located in exon 12 was used (Figure $4 b$, top panel).

Earlier reports have identified a splice variant of lamin A that lacked exon 10 , lamin $\mathrm{A} \Delta 10 .^{8}$ This splice form was detected in our cDNA samples when using a forward primer spanning the junction of exons 9 and 11, and a reverse primer in exon 12 (Figure $4 \mathrm{~b}$, second panel). This same primer pair also amplified a shorter fragment in HGPS samples, which showed that both lamin A and A $\Delta 150$ transcripts have alternative splice forms that lack exon 10.

\section{Quantification of $L M N A$ protein isoforms}

Protein extracts from different passages of primary fibroblast cultures from HGPS patients and unaffected controls were analyzed using western blot, with antibodies to human lamin A/C (Figure 4c). All protein quantifications were performed on western blots hybridized to the human lamin A/C antibody, mab3211 (Figure 4c). Similar results were also seen with an N-terminal lamin A/C antibody, N-18 (data not shown). Samples were normalized to $\beta$-actin. Quantification of lamin A included the band densities for proteins corresponding in size to prelamin A and lamin A (Figure 4c). Quantification of lamin C included the band densities for the two fragments recognized by the lamin C-specific antibody and that corresponded to lamin $\mathrm{C}$ in size. A fragment corresponding in size to progerin was only seen in samples from HGPS patients (Figure $4 \mathrm{a}$ and c, and data not shown). To analyze changes in lamins $\mathrm{A}, \mathrm{C}$, and progerin proteins during in vitro cell aging, the normalized band densities from late passage cells were divided by the normalized band densities from early passage cells of the same cell culture. If the obtained ratio was higher than 1, the protein expression had increased with passaging, but if the ratio was lower than 1, the protein expression had decreased with passaging.

Analysis of changes in lamins A, C, and progerin protein levels with aging in HGPS cells, did not show a statistical significant difference to 1 for any of the proteins (mean values were $1.2(P=0.586), 0.9 \quad(P=0.741)$, and 1.3 $(P=0.458)$ for lamins $\mathrm{A}, \mathrm{C}$, and progerin, respectively; Figure $4 \mathrm{c}$ and $\mathrm{d})$. Similarly, there was no significant increase in lamins $\mathrm{A}$ and $\mathrm{C}$ protein levels with aging in unaffected control cells (sample group included samples from both age-matched and parents; mean values were 1.9 $(P=0.318)$ and $1.3(P=0.363)$, respectively). In addition, large standard deviations showed that all sample groups contained samples in which protein levels had both increased and decreased with passage number (Figure $4 \mathrm{c}$ and d). There was no significant difference between the mean ratios of lamin A or $\mathrm{C}$ when comparing the HGPS and unaffected control group $(P=0.494$ and $P=0.339$, respectively; Figure 4d).

\section{Discussion}

Determination of the overall expression levels of the $L M N A$ locus transcripts is an important step in further understanding the molecular mechanisms of the HGPS. Existing data on the expression of the LMNA gene in HGPS are based on semiquantitative RT-PCR techniques, or there is a lack of data on the expression of lamin C. ${ }^{20,21,28}$ In this study, we have developed a method for absolute quantification of the lamins A, C, and A $\Delta 150$ transcripts. Absolute quantification of these transcripts during in vitro aging of HGPS patient cell cultures and unaffected controls showed different expression levels and regulation. To analyze normal changes in the LMNA gene expression during aging, samples from two differently aged normal control groups were included. Our results showed that lamin $\mathrm{C}$ is the most highly expressed LMNA locus transcript, in both HGPS and control groups. Considering that lamins A and C are regulated by the same promoter, our results would suggest that the splicing efficiency for the lamin $\mathrm{C}$ transcript was favored and/or the lamin $\mathrm{C}$ transcripts were more stable. Lamin $C$ transcripts were significantly higher in the unaffected parent control samples when compared with those in samples from HGPS. However, as there was no significant difference in lamin $\mathrm{C}$ expression between HGPS and age-matched controls, or between the two control groups, these results are hard to interpret.

In accordance with earlier studies, ${ }^{20,21}$ we have shown that the lamin $\mathrm{A} \Delta 150$ transcript is present in cells from normal individuals, in addition to cells from HGPS patients. Our results showed that the lamin $\mathrm{A} \Delta 150$ transcript level was >160-fold higher in samples from HGPS compared with that in the unaffected controls. An earlier study has reported a 50-fold lower expression of the lamin A $\Delta 150$ transcript in normal cells compared with that in HGPS cells. ${ }^{20}$ The difference in the results could be a result of differences in methodology or by variations in progerin mRNA levels between HGPS patients. ${ }^{28}$

In this study, we have shown that lamin $\mathrm{A} \Delta 150$ transcript levels in HGPS cells are present at the same levels as lamin A transcripts in unaffected controls. This is in agreement with findings from an earlier study, ${ }^{29}$ which showed that both the wild-type and mutant alleles in HGPS patients are capable of comparable levels of transcription. Perhaps a surprising finding in this study was that the presence of lamin A $\Delta 150$ transcripts in HGPS cells did not affect the normal transcript levels of lamin A. Absolute quantification of the LMNA locus transcripts in HGPS cells showed that the combined amounts of lamins A and $\mathrm{A} \Delta 150$ are about twice the amount compared with what are found in normal controls (Figure 2). This might be explained by differences in mRNA stability and accumulation of the lamin A $\Delta 150$ transcript, or increased LMNA gene expression in HGPS cells. Given that both the lamins $\mathrm{C}$ and A transcript copy numbers in HGPS cells were similar to what was seen in unaffected age-matched control 
samples, an upregulation of $L M N A$ gene expression would then have to be combined with a decreased splicing to form lamin $\mathrm{C}$, and/or increased splicing to form lamins A and $\mathrm{A} \Delta 150$.

Our quantification of lamins $\mathrm{A}, \mathrm{C}$ and $\mathrm{A} \Delta 150$ transcripts in RNA from different passages of cells showed that although the lamin A and C transcripts were unchanged, the lamin $\mathrm{A} \Delta 150$ transcript levels increased in both late passage HGPS and unaffected parent control cells compared with that in early passage cells. These results might indicate a similar mechanism in progeria and normal aging cells with a reduced stringency of the splicing machinery with aging. Cells from normal younger individuals are somehow protected, and therefore less prone to abnormal splicing during in vitro aging. However, in this study and in agreement with earlier reports, using a different experimental approach, ${ }^{20,21}$ there was no difference in the lamin A $\Delta 150$ transcripts levels between differently aged normal control samples (Figure $2 \mathrm{~b}$ ), which argues against age as a significant modifier of lamin A $\Delta 150$ expression. Future studies with larger control sample sets and perhaps with samples from older individuals might relate our results to in vivo cellular aging. ${ }^{30}$

Extensive RT-PCR analysis of novel LMNA splicing did not reveal any alternative splice forms, which suggests that the two lamin $\mathrm{C}$ fragments, seen in our samples using enhanced protein separation, migrate differently because of differences in post-translational modification. Our results emphasize the importance of using larger gels or high separation when analyzing proteins of similar molecular weights.

In contrast with earlier studies on HGPS cells, ${ }^{14,31}$ we have, in this study, shown that the amount of progerin protein does not increase with passaging. This is in agreement with a recent study from Verstraeten et $a l^{32}$ where progerin levels remained constant during passaging of HGPS cells. It is unclear whether the differences between the different studies reflect actual inter-sample variations of progerin or is a consequence of differences in experimental techniques. In our relative quantification of lamins A, C, and progerin proteins in early and late passages, we have seen varied results within the sample groups, which are reflected by extensive standard deviations. This would argue in favor of inter-sample variation of protein levels.

In summary, we have developed a method for absolute quantification of LMNA locus transcripts, which should be useful for future studies on HGPS, laminopathies, and lamins involvement in physiological aging.

\section{Acknowledgements}

We thank Mubashir Hanif for his expert technical assistance, and Karin Dillner and Caroline Graff for their helpful discussions on assay design. This study was supported by grants from the Gun and Bertil Stohne Foundation, the Ake Wiberg Foundation, the Magnus Bergvall Foundation, the Tore Nilsson Foundation, the Loo and Hans Osterman
Foundation, the Torsten and Ragnar Söderberg Foundations, the Jeansson Foundations, the Swedish Medical Research Council and the Swedish Foundation for Strategic Research.

\section{Conflict of interest}

None.

\section{References}

1 Martin GM: Genetic modulation of senescent phenotypes in Homo sapiens. Cell 2005; 120: 523-532.

2 Pollex RL, Hegele RA: Hutchinson-Gilford progeria syndrome. Clin Genet 2004; 66: 375-381.

3 De Sandre-Giovannoli A, Bernard R, Cau P et al: Lamin a truncation in Hutchinson-Gilford progeria. Science 2003; 300: 2055.

4 Eriksson M, Brown WT, Gordon LB et al: Recurrent de novo point mutations in lamin A cause Hutchinson-Gilford progeria syndrome. Nature 2003; 423: 293-298.

5 Goldman RD, Gruenbaum Y, Moir RD, Shumaker DK, Spann TP: Nuclear lamins: building blocks of nuclear architecture. Genes Dev 2002; 16: 533-547.

6 Fisher DZ, Chaudhary N, Blobel G: cDNA sequencing of nuclear lamins $\mathrm{A}$ and $\mathrm{C}$ reveals primary and secondary structural homology to intermediate filament proteins. Proc Natl Acad Sci USA 1986; 83: 6450-6454.

7 Rober RA, Weber K, Osborn M: Differential timing of nuclear lamin A/C expression in the various organs of the mouse embryo and the young animal: a developmental study. Development 1989; 105: $365-378$.

8 Machiels BM, Zorenc AH, Endert JM: An alternative splicing product of the lamin A/C gene lacks exon 10. J Biol Chem 1996; 271: 9249-9253.

9 Furukawa K, Inagaki H, Hotta Y: Identification and cloning of an mRNA coding for a germ cell-specific A-type lamin in mice. Exp Cell Res 1994; 212: 426-430.

10 Beck LA, Hosick TJ, Sinensky M: Isoprenylation is required for the processing of the lamin A precursor. J Cell Biol 1990; 110: $1489-1499$.

11 Capell BC, Collins FS: Human laminopathies: nuclei gone genetically awry. Nat Rev Genet 2006; 7: 940-952.

12 Sinensky M, Fantle K, Trujillo M, McLain T, Kupfer A, Dalton M The processing pathway of prelamin A. J Cell Sci 1994; 107: 61-67.

13 Dechat T, Shimi T, Adam SA et al: Alterations in mitosis and cell cycle progression caused by a mutant lamin A known to accelerate human aging. Proc Natl Acad Sci USA 2006; 104: $4955-4960$.

14 Goldman RD, Shumaker DK, Erdos MR et al: Accumulation of mutant lamin A causes progressive changes in nuclear architecture in Hutchinson-Gilford progeria syndrome. Proc Natl Acad Sci USA 2004; 101: 8963-8968.

15 Shumaker DK, Dechat T, Kohlmaier A et al: Mutant nuclear lamin A leads to progressive alterations of epigenetic control in premature aging. Proc Natl Acad Sci USA 2006; 103: $8703-8708$

16 Ly DH, Lockhart DJ, Lerner RA, Schultz PG: Mitotic misregulation and human aging. Science 2000; 287: 2486-2492.

17 Csoka AB, English SB, Simkevich CP et al: Genome-scale expression profiling of Hutchinson-Gilford progeria syndrome reveals widespread transcriptional misregulation leading to mesodermal/mesenchymal defects and accelerated atherosclerosis. Aging Cell 2004; 3: 235-243.

18 Cao K, Capell BC, Erdos MR, Djabali K, Collins FS: A lamin A protein isoform overexpressed in Hutchinson-Gilford progeria syndrome interferes with mitosis in progeria and normal cells. Proc Natl Acad Sci USA 2007; 104: 4949-4954. 
19 Haithcock E, Dayani Y, Neufeld E et al: Age-related changes of nuclear architecture in Caenorhabditis elegans. Proc Natl Acad Sci USA 2005; 102: 16690-16695.

20 Scaffidi P, Misteli T: Lamin A-dependent nuclear defects in human aging. Science 2006; 312: 1059-1063.

21 McClintock D, Ratner D, Lokuge $\mathrm{M}$ et al: The mutant form of lamin A that causes Hutchinson-Gilford progeria is a biomarker of cellular aging in human skin. PLoS ONE 2007; 2: e1269.

22 Hayflick L: The limited in vitro lifetime of human diploid cell strains. Exp Cell Res 1965; 37: 614-636.

23 Brown WT: Genetic diseases of premature aging as models of senescence. Annu Rev Gerontol Geriatr 1990; 10: 23-42.

24 Rozen S, Skaletsky H: Primer3 on the WWW for general users and for biologist programmers. Methods Mol Biol 2000; 132: $365-386$

25 Eriksson M: Real-time RT-PCR for CTG repeat-containing genes. Methods Mol Biol 2004; 277: 77-84.

26 Sagelius H, Rosengardten Y, Hanif $M$ et al: Targeted transgenic expression of the mutation causing Hutchinson-Gilford progeria syndrome leads to proliferative and degenerative epidermal disease. J Cell Sci 2008; 121: 969-978.
27 Nagasaka Y, Dillner K, Ebise $\mathrm{H}$ et al: A unique gene expression signature discriminates familial Alzheimer's disease mutation carriers from their wild-type siblings. Proc Natl Acad Sci USA 2005; 102: $14854-14859$.

28 Moulson CL, Fong LG, Gardner JM et al: Increased progerin expression associated with unusual LMNA mutations causes severe progeroid syndromes. Hum Mutat 2007; 28: 882-889.

29 Reddel CJ, Weiss AS: Lamin A expression levels are unperturbed at the normal and mutant alleles but display partial splice site selection in Hutchinson-Gilford progeria syndrome. J Med Genet 2004; 41: 715-717

30 Reff M, Schneider EL: Cell culture aging. Mol Cell Biochem 1981; 36: $169-176$.

31 McClintock D, Gordon LB, Djabali K: Hutchinson-Gilford progeria mutant lamin A primarily targets human vascular cells as detected by an anti-lamin A G608G antibody. Proc Natl Acad Sci USA 2006; 103: 2154-2159.

32 Verstraeten VL, Ji JY, Cummings KS, Lee RT, Lammerding J: Increased mechanosensitivity and nuclear stiffness in Hutchinson-Gilford progeria cells: effects of farnesyltransferase inhibitors. Aging Cell 2008; 7: 383-393. 